\title{
Near-horizon of 5D rotating black holes from 2D perspective
}

\author{
Hesam Soltanpanahi ${ }^{\mathrm{a}}$ \\ Institute of Physics, Jagiellonian University, Reymonta 4, 30-059 Kraków, Poland
}

Received: 28 December 2013 / Accepted: 6 March 2014 / Published online: 20 March 2014

(C) The Author(s) 2014. This article is published with open access at Springerlink.com

\begin{abstract}
We study the CFT dual to five-dimensional extremal rotating black holes, by investigating the twodimensional perspective of their near-horizon geometry. From the two-dimensional point of view, we show that both gauge fields, related to the two rotations, appear in the same manner in the asymptotic symmetry and in the associated central charge. We find that our results are in perfect agreement with the generalization of the Kerr/CFT approach to five-dimensional extremal rotating black holes.
\end{abstract}

\section{Introduction}

Five-dimensional black holes have been interesting ever since the seminal work on computing the entropy of a 5D black hole by Strominger and Vafa in the context of string theory [1]. Recently by extending the Kerr/CFT [2] approach to $5 \mathrm{D}$ extremal rotating black holes (ERBH) a wide class of such solutions have been studied [3-11]. A common feature of these studies is the appearance of two rotating coordinates in the near-horizon geometry of most of these solutions as well as an $\mathrm{AdS}_{2}$ part. It was proposed in some of these works that there are two dual CFTs, each of which corresponds to one of the rotations.

It was shown in [10] that these two CFTs are related to each other by the $\operatorname{SL}(2, \mathbb{Z})$ transformation which is a symmetry in the space of the moduli parameters of the near-horizon geometry of the 5D black holes with two rotating coordinates. For this propose the boundary conditions for the rotating coordinates are in the same order such that the symmetry of the rotating coordinates is preserved by boundary fluctuations. This is not the case for the 5D black holes with only one rotation, e.g. black ring.

In this note we show that the consistency of the boundary conditions from the $2 \mathrm{D}$ point of view requires that the two gauge fields should be treated on the same footing in the study

\footnotetext{
a e-mail: hesam@th.if.uj.edu.pl
}

of an asymptotic symmetry group. This leads to a chiral CFT, with a central special central charge, corresponding to the near-horizon geometry of 5D extremal double rotating black holes.

Each of the rotating coordinates in 5D reduces to one of the gauge fields from the $2 \mathrm{D}$ perspective. By introducing proper boundary conditions for the gauge fields and for the boundary energy-momentum tensor we calculate the corresponding central charge of the dual CFT. This approach resembles the quantum entropy function introduced by Ashoke Sen [12], where all of the gauge fields are considered in the same manner to study the thermodynamics of extremal black holes.

By using the approach introduced by Castro and Larsen [13], we reduce the near-horizon geometry of 5D extremal double rotating black holes to a $2 \mathrm{D}$ theory and investigate the properties of the boundary energy-momentum tensor of the $\mathrm{AdS}_{2}$ metric. We show that the variation of the energymomentum tensor under diffeomorphism which should be combined with gauge transformations [14] admits one central charge. As an example we calculate the associated central charge for the Myers-Perry black hole [15] and show the agreement with known results in this case.

The remainder of this paper is organized as follows. In Sect. 2 we briefly review the 5D extremal rotating black hole and its CFT dual from the 5D point of view. In Sect. 3, following [13], we study the reduction of 5D extremal rotating black hole to the AdS solution of 2D theory. Then we derive the boundary terms of the $2 \mathrm{D}$ action and investigate the consistency of the boundary conditions which are allowed for 2D theory. By using the notion of the Peierls bracket [16] and the counter-term subtraction charge [17] we define the associated charge and compute the central charge associated to the variation of the boundary energy momentum tensor in Sect. 4. In Sect. 5 we study the Myers-Perry black hole with two rotations and we show the agreement of our results with the previous calculations. Finally, Sect. 6 contains our conclusions and a brief discussion. 


\section{Review of 5D ERBH/CFT}

This section is devoted to a review of the generalization of Kerr/CFT approach for 5D extremal rotating black holes. The reader who is familiar with the Kerr/CFT approach can skip this section. We wil mention the main steps of the calculations and will not discuss the details, which can be found in [10].

The near-horizon geometry of 5D ERBH is given by [18$20]^{1}$

$$
\begin{aligned}
\mathrm{d} s_{5}^{2}= & F(\theta) \mathrm{d} s_{\mathrm{AdS} S_{2}}^{2}+\sigma(\theta) \mathrm{d} \theta^{2} \\
& +\gamma_{i j}(\theta)\left(\mathrm{d} x^{i}+k^{i} r \mathrm{~d} t\right)\left(\mathrm{d} x^{j}+k^{j} r \mathrm{~d} t\right) .
\end{aligned}
$$

Possible boundary conditions for the fluctuations around this geometry (1) are [10]

$h_{\mu \nu} \sim \mathcal{O}\left(\begin{array}{lllll}r^{2} & 1 / r^{2} & 1 / r & r & r \\ & 1 / r^{3} & 1 / r^{2} & 1 / r & 1 / r \\ & & 1 / r & 1 / r & 1 / r \\ & & & 1 & 1 \\ & & & & 1\end{array}\right)$,

in the basis $\left(t, r, \theta, \phi_{1}, \phi_{2}\right)$. These boundary conditions are consistent with the symmetry of the near-horizon geometry which combine the $\phi_{1,2}$ coordinates with each other.

A general diffeomorphism preserving the boundary conditions (2) is given by

$$
\begin{aligned}
\zeta= & {\left[C+\mathcal{O}\left(\frac{1}{r^{3}}\right)\right] \partial_{t}+\left[r \epsilon\left(\phi_{1}, \phi_{2}\right)+\mathcal{O}(1)\right] \partial_{r} } \\
& +\mathcal{O}\left(\frac{1}{r}\right) \partial_{\theta}+\left[\lambda_{1}\left(\phi_{1}, \phi_{2}\right)+\mathcal{O}\left(\frac{1}{r^{2}}\right)\right] \partial_{\phi_{1}} \\
& +\left[\lambda_{2}\left(\phi_{1}, \phi_{2}\right)+\mathcal{O}\left(\frac{1}{r^{2}}\right)\right] \partial_{\phi_{2}},
\end{aligned}
$$

where $\epsilon\left(\phi_{1}, \phi_{2}\right)$ and $\lambda_{1,2}\left(\phi_{1}, \phi_{2}\right)$ are arbitrary smooth periodic functions of $\phi_{1}$ and $\phi_{2}$.

In [10] it was shown that the class of diffeomorphism generators has basis

$$
\begin{aligned}
\zeta_{m}= & -\mathrm{e}^{-i m \phi_{1}} \partial_{\phi_{1}}-\mathrm{e}^{-i m \phi_{2}} \partial_{\phi_{2}} \\
& -2 i m r\left(\mathrm{e}^{-i m \phi_{1}}+\mathrm{e}^{-i m \phi_{2}}\right) \partial_{r},
\end{aligned}
$$

and they satisfy the Virasoro algebra $\left[\zeta_{m}, \zeta_{n}\right]_{\text {Lie }}=-i(m-$ n) $\zeta_{m+n}$. These generators correspond to a chiral $\mathrm{CFT}_{2}$.

Using the definition of diffeomorphism charges [21,22] and following the Brown-Henneaux approach [23], it was shown that there is a Virasoro algebra between the associated charges with the central charge which is given by

$c=\frac{3\left(k_{1}+k_{2}\right)}{2 \pi} \int \mathrm{d} \theta \mathrm{d} \phi_{1} \mathrm{~d} \phi_{2} \sqrt{\sigma(\theta) \gamma(\theta)}$.

${ }_{1}$ The $\mathrm{AdS}_{2}$ radius has been absorbed in $F(\theta)$.
We want to emphasize that both of the $k_{1,2}$, which correspond to the angular momenta, contribute in the value of central charge in (5).

In the next two sections we confirm this result from the 2D perspective. For this propose we reduce the 5D near-horizon geometry (1) to $2 \mathrm{D}$ theory by integrating out the angular coordinates. The resulting solution has an $\mathrm{AdS}_{2}$ metric and two gauge fields related to two angular momenta. We show that the combination of the diffeomorphism and gauge transformations of both of the gauge fields is consistent for investigating the variation of the boundary energy-momentum tensor. In Sect. 5 we show the agreement of $2 \mathrm{D}$ results with the 5D results for Myers-Perry black holes.

\section{2D view of 5D extremal rotating black holes}

In this section, we want to study the 5D ERBH from the 2D perspective. The next section is devoted to a calculation of the conserved charges and central charge following Castro and Larsen [13]. The steps and arguments are similar to [13], so we do not give all the details. By using the reduction we will show that both of the gauge fields in 2D, associated to two rotating coordinates, play the same role in studying the asymptotic symmetry and the AdS/CFT correspondence. This is $2 \mathrm{D}$ evidence for the arguments reviewed in Sect. 2.

\subsection{D ERBH}

We start with the general form of the near-horizon geometry of 5D ERBH with two angular momenta and by reduction on angular coordinates we obtain a $2 \mathrm{D}$ effective theory. As we mentioned in (1), the near-horizon geometry of 5D ERBH with two rotations is given by

$$
\begin{aligned}
\mathrm{d} s_{5}^{2}= & F(\theta) \mathrm{d} s_{\mathrm{AdS}}^{2}+\sigma(\theta) \mathrm{d} \theta^{2} \\
& +\gamma_{i j}(\theta)\left(\mathrm{d} x^{i}+k^{i} r \mathrm{~d} t\right)\left(\mathrm{d} x^{j}+k^{j} r \mathrm{~d} t\right),
\end{aligned}
$$

where $F(\theta), \sigma(\theta)$ and $\gamma_{i j}(\theta)$ are the functions of only $\theta$ and $x^{i}, i=1,2$ correspond to the rotating coordinates.

By integrating out the angular part, the two-dimensional theory could be described by a general 2D metric

$\mathrm{d} s^{2}=g_{\mu \nu} \mathrm{d} x^{\mu} \mathrm{d} x^{\nu}$,

and two gauge fields corresponding to the rotations,

$\mathcal{A}^{i}=\mathcal{A}_{\mu}^{i} \mathrm{~d} x^{\mu}, \quad i=1,2$,

with $\mu, v=t, r$. We also couple the size of the angular coordinates to the scalar field $\psi$ such that

$$
\begin{aligned}
\mathrm{d} s^{2}= & F(\theta) \mathrm{d} s_{2}^{2}+\mathrm{e}^{-2 \psi}\left[\sigma(\theta) \mathrm{d} \theta^{2}\right. \\
& \left.+\gamma_{i j}(\theta)\left(\mathrm{d} x^{i}+\mathcal{A}^{i}\right)\left(\mathrm{d} x^{j}+\mathcal{A}^{j}\right)\right] .
\end{aligned}
$$


Lowering and raising the indices are defined by $g_{\mu \nu}$ and its inverse $g^{\mu \nu}$, respectively. The associated gauge field strengths are denoted by $\mathcal{F}^{i}=\mathrm{d} \mathcal{A}^{i}$.

The 5D Einstein Hilbert action is

$$
S_{(5)}=\frac{1}{16 \pi G_{5}} \int \mathrm{d}^{5} x \sqrt{-g_{5}} R^{(5)} \text {. }
$$

By using the ansatz (9), one can find the five-dimensional Ricci scalar from a two-dimensional point of view, ${ }^{2}$

$$
\begin{aligned}
R^{(5)}= & \frac{1}{F(\theta)}\left[R^{(2)}-3 \mathrm{e}^{2 \psi} \nabla^{2} \mathrm{e}^{-2 \psi}\right] \\
& -\frac{\mathrm{e}^{-2 \psi}}{F(\theta)^{2}} \gamma_{i j}(\theta) \mathcal{F}_{\mu \nu}^{i} F^{j, \mu \nu}+H(\theta) \mathrm{e}^{2 \psi},
\end{aligned}
$$

in which

$$
\begin{aligned}
H(\theta)= & \frac{1}{\sigma}\left\{\frac{1}{2}\left(\frac{\mathrm{d}}{\mathrm{d} \theta} \ln \frac{F}{\gamma}\right)^{2}-\frac{2}{F}\left(\frac{\mathrm{d}^{2} F}{\mathrm{~d} \theta^{2}}\right)\right. \\
& -\frac{3}{2 \gamma} \operatorname{det}\left|\frac{\mathrm{d}}{\mathrm{d} \theta} \gamma_{i j}\right|+\left(\frac{\mathrm{d}}{\mathrm{d} \theta} \ln F \gamma\right)\left(\frac{\mathrm{d}}{\mathrm{d} \theta} \ln \sigma\right) \\
& -\frac{1}{f}\left(\frac{\mathrm{d}}{\mathrm{d} \theta} \ln \sigma\right)\left[\gamma_{\phi \phi}\left(\frac{\mathrm{d}^{2} \gamma_{\psi \psi}}{\mathrm{d} \theta^{2}}\right)\right. \\
& \left.\left.+\gamma_{\psi \psi}\left(\frac{\mathrm{d}^{2} \gamma_{\phi \phi}}{\mathrm{d} \theta^{2}}\right)-2 \gamma_{\phi \psi}\left(\frac{\mathrm{d}^{2} \gamma_{\phi \psi}}{\mathrm{d} \theta^{2}}\right)\right]\right\},
\end{aligned}
$$

and the five-dimensional determinant is

$\sqrt{-g_{5}}=\mathrm{e}^{-3 \psi} \sqrt{F(\theta)^{2} \sigma(\theta) \gamma(\theta)} \sqrt{-g}$,

where

$$
\gamma(\theta) \equiv \operatorname{det}\left|\gamma_{i j}(\theta)\right| \text {. }
$$

By integrating over the angular coordinates, the $2 \mathrm{D}$ effective action of ERBH can be derived as

$$
\begin{aligned}
S_{(2)}= & \frac{\pi \alpha}{4 G_{5}} \int \mathrm{d}^{2} x \sqrt{-g}\left[\mathrm{e}^{-3 \psi} R^{(2)}+\beta \mathrm{e}^{-\psi}\right. \\
& \left.+\frac{8}{3} \nabla_{\mu} \mathrm{e}^{-\frac{3}{2} \psi} \nabla^{\mu} \mathrm{e}^{-\frac{3}{2} \psi}-M_{i j} \mathcal{F}_{\mu \nu}^{i} F^{j, \mu \nu} \mathrm{e}^{-5 \psi}\right],
\end{aligned}
$$

in which

$\alpha=\int \mathrm{d} \theta \sqrt{\sigma(\theta) \gamma(\theta)}$,

$\beta=\frac{1}{\alpha} \int \mathrm{d} \theta \sqrt{F(\theta)^{2} \sigma(\theta) \gamma(\theta)} H(\theta)$,

$M_{i j}=\frac{1}{\alpha} \int \mathrm{d} \theta \frac{\sqrt{F(\theta)^{2} \sigma(\theta) \gamma(\theta)}}{F(\theta)^{2}} \gamma_{i j}(\theta)$.

The action (15) might be considered as the generic dilaton gravity in 2D with two gauge fields which was introduced in $[24,25]$.

\footnotetext{
${ }^{2}$ We use indices, $\mu, v$ for coordinates $r, t$, and the indices $i, j$ for the gauge fields.
}

\subsection{Solutions}

Since we are interested in solutions corresponding to the geometry (6), we limit ourselves to the solutions with constant $\psi$ and we try to solve the following equations of motion:

$3 R^{(2)} \mathrm{e}^{-2 \psi}-5 M_{i j} \mathcal{F}_{\mu \nu}^{i} F^{j, \mu \nu} \mathrm{e}^{-4 \psi}+\beta=0$,

$\frac{1}{2}\left(\beta-M_{i j} \mathcal{F}_{\rho \tau}^{i} F^{j, \rho \tau} \mathrm{e}^{-4 \psi}\right) g_{\mu \nu}$

$$
\begin{aligned}
& \quad+2 M_{i j} \mathcal{F}_{\mu \rho}^{i} \mathcal{F}_{\nu}^{j, \rho} \mathrm{e}^{-4 \psi}=0, \\
& \nabla_{\mu} F^{i, \mu \nu}=0 .
\end{aligned}
$$

The first and second equations can be simplified to

$M_{i j} \mathcal{F}_{\mu \nu}^{i} F^{j, \mu \nu}=-\beta \mathrm{e}^{4 \psi}$

$R^{(2)}=-2 \beta \mathrm{e}^{2 \psi}$.

Assuming $\beta>0$, which is natural for the reduction of an extremal solution over angular coordinates, this solution is locally $\mathrm{AdS}_{2}$ with radius

$l_{\text {AdS }}=\left(\frac{1}{\beta}\right)^{1 / 2} \mathrm{e}^{-\psi} \equiv l \mathrm{e}^{-\psi}$.

As one can see from (17) $l_{\text {AdS }}$ is dimensionless. It is because we absorb the radius of the $\mathrm{AdS}_{2}$ part of the near-horizon geometry (6) in $F(\theta)$.

Without losing generality, we work in the gauge

$\mathrm{d} s^{2}=\mathrm{e}^{-2 \psi} \mathrm{d} \rho^{2}+g_{t t} \mathrm{~d} t^{2}, \quad \mathcal{A}_{\mu}^{i} \mathrm{~d} x^{\mu}=\mathcal{A}_{t}^{i}(\rho, t) \mathrm{d} t$.

In this gauge, the general form of the solution of equations of motion is given by

$g_{t t}=-\frac{1}{4} \mathrm{e}^{-2 \psi}\left(\mathrm{e}^{\rho / l}-f(t) \mathrm{e}^{-\rho / l}\right)^{2}$

$\mathcal{A}_{t}^{i}=\frac{\lambda^{i}}{2 l} \mathrm{e}^{\rho / l}\left(1-\sqrt{f(t)} \mathrm{e}^{-\rho / l}\right)^{2}$,

with the constraint

$M_{i j} \lambda^{i} \lambda^{j}=\frac{l^{2}}{2}$.

Note that the constants $\lambda_{i} \neq 0$ are inherited from $k_{i} \neq 0$, which means the near-horizon geometry of 5D ERBH (6) has two non-zero angular momenta.

It is convenient to describe this solution in the FeffermanGraham coordinate expansion. The asymptotic behavior of the metric, scalar, and gauge fields are given, respectively, by

$g_{t t}^{(0)}=-\frac{1}{4} \mathrm{e}^{-2 \psi^{(0)}} \mathrm{e}^{2 \rho / l}$,

$\mathcal{A}_{t}^{i(0)}=\frac{\lambda^{i}}{2 l} \mathrm{e}^{\rho / l}$,

$\psi^{(0)}=$ constant.

The result is similar to the reduction of the four-dimensional extremal Kerr black hole studied in [13,26]. 


\subsection{Boundary terms}

In this section, following the standard procedure for AdS/CFT we determine the normalized boundary action which is formally given by

$S_{\text {boundary }}=S_{\mathrm{GHY}}+S_{\text {counter }}$.

The first term is the Gibbons-Hawking-York term, namely

$S_{\mathrm{GHY}}=\frac{2 \pi \alpha}{4 G_{5}} \int_{\partial \mathcal{M}} \mathrm{d} t \sqrt{-h} \mathrm{e}^{-3 \psi} K$

where $h$ and $K$ are, respectively, the determinant of the induced metric and the extrinsic curvature on the boundary $\partial \mathcal{M}$. It is easy to show that for solution (27) the extrinsic curvature is

$K=\frac{1}{2} g^{t t} n^{\mu} \partial_{\mu} g_{t t}=\frac{1}{l} \mathrm{e}^{\psi}$.

As discussed in $[13,26]$ the local form of the counter-term is given by

$$
\begin{aligned}
S_{\text {counter }}= & \frac{2 \pi \alpha}{4 G_{5}} \int_{\partial \mathcal{M}} \mathrm{d} t \sqrt{-h} \\
& \times\left[m_{1} \mathrm{e}^{-2 \psi}+m_{2} \mathrm{e}^{-4 \psi} M_{i j} \mathcal{A}_{a}^{i} \mathcal{A}^{j, a}\right],
\end{aligned}
$$

in which the constants $m_{1}$ and $m_{2}$ will be determined by vanishing of the variation of the action on-shell.

On the other hand, the variation of the action is given by $\delta S=\int_{\partial \mathcal{M}}\left[\pi^{a b} \delta h_{a b}+\pi_{\psi} \delta \psi+\pi_{i}^{a} \delta \mathcal{A}_{a}^{i}\right]+$ Bulk terms

with

$$
\begin{aligned}
\pi^{t t} & =\frac{\pi \alpha}{4 G_{5}}\left(m_{1} \mathrm{e}^{-2 \psi} h^{t t}+m_{2} \mathrm{e}^{-4 \psi} h^{t t} M_{i j} \mathcal{A}_{\mu}^{i} \mathcal{A}^{j, \mu}\right. \\
& \left.-2 m_{2} \mathrm{e}^{-4 \psi} M_{i j} \mathcal{A}^{i, t} \mathcal{A}^{j, t}\right), \\
\pi_{\psi}= & \frac{2 \pi \alpha}{4 G_{5}}\left(-3 \mathrm{e}^{-3 \psi} K-2 m_{1} \mathrm{e}^{-2 \psi}-4 m_{2} \mathrm{e}^{-4 \psi} M_{i j} \mathcal{A}_{a}^{i} \mathcal{A}^{j, a}\right), \\
\pi_{i}^{t}= & \frac{\pi \alpha}{4 G_{5}}\left(-4 \mathrm{e}^{-5 \psi} M_{i j} n_{\mu} \mathcal{F}^{j, \mu t}+4 m_{2} \mathrm{e}^{-3 \psi} M_{i j} \mathcal{A}^{j, t}\right) .
\end{aligned}
$$

Using the asymptotic behavior of the fields (29) and the extrinsic curvature (32), the above expansions for conjugate momenta are reduced to

$$
\begin{aligned}
\pi^{t t} & =\frac{\pi \alpha}{4 G_{5}}\left(m_{1}+\frac{m_{2}}{2}\right) \mathrm{e}^{-2 \psi^{(0)}} h_{(0)}^{t t}, \\
\pi_{\psi} & =-\frac{2 \pi \alpha}{4 G_{5}}\left(\frac{3}{l}+2 m_{1}-2 m_{2}\right) \mathrm{e}^{-2 \psi^{(0)}}, \\
\pi_{i}^{t} & =\frac{2 \pi \alpha}{4 G_{5}}\left(-1+m_{2} l\right) M_{i j} \lambda^{j} \mathrm{e}^{-4 \psi^{(0)}} h_{(0)}^{t t} \mathrm{e}^{\rho / l} .
\end{aligned}
$$

One can fix the constants $m_{1,2}$ by imposing vanishing boundary momenta (38), which leads to two conditions,

$m_{1}=-\frac{1}{2 l}, \quad m_{2}=\frac{1}{l}$

Note that, although there were two unknown constants $m_{1,2}$, we had three equations; therefore, finding a solution shows the consistency of our calculations. In this way the full action of reduced solution is given by

$$
\begin{aligned}
S= & \frac{\pi \alpha}{4 G_{5}} \int_{\mathcal{M}} \mathrm{d}^{2} x \sqrt{-g}\left[\mathrm{e}^{-3 \psi} R^{(2)}\right. \\
& \left.+\beta \mathrm{e}^{-\psi}+\frac{4}{3} \nabla_{\mu} \mathrm{e}^{-\frac{3}{2} \psi} \nabla^{\mu} \mathrm{e}^{-\frac{3}{2} \psi}-M_{i j} \mathcal{F}_{\mu \nu}^{i} F^{j, \mu \nu} \mathrm{e}^{-5 \psi}\right] \\
& +\frac{\pi \alpha}{2 G_{5}} \int_{\partial \mathcal{M}} \mathrm{d} t \sqrt{-h}\left[\mathrm{e}^{-3 \psi} K-\frac{1}{2 l} \mathrm{e}^{-2 \psi}\right. \\
& \left.+\frac{1}{l} \mathrm{e}^{-4 \psi} M_{i j} \mathcal{A}_{a}^{i} \mathcal{A}^{j, a}\right] .
\end{aligned}
$$

\subsection{Consistency of boundary conditions}

As discussed in [14,26], for the AdS solution with a gauge field the combination of diffeomorphism and gauge transformation should be consistent with the gauge conditions. In this section we show that for the solution (27), i.e. the AdS metric with two gauge fields, the consistency requires that the gauge transformations of both of the gauge fields should be included in addition to the diffeomorphism. For this purpose we first determine the diffeomorphism of the metric and its consequences for the gauge fields. Then we find the compensating gauge transformations leaving the gauge fields in the gauge condition (25).

The general diffeomorphism transforms the metric as

$\delta_{\epsilon} g_{\mu \nu}=\nabla_{\mu} \epsilon_{\nu}+\nabla_{\nu} \epsilon_{\mu}$.

The gauge condition (25) has fixed the $g_{\rho \rho}$ and $g_{t \rho}$ components of the metric to zero and by using the FeffermanGraham form we have fixed the asymptotic value of the $g_{t t}$ (29). Thus one can find the associated diffeomorphism which preserves these conditions by requiring the following conditions:

$\delta_{\epsilon} g_{\rho \rho}=0, \quad \delta_{\epsilon} g_{t \rho}=0, \quad \delta_{\epsilon} g_{t t}=0 . \mathcal{O}\left(\mathrm{e}^{2 \rho / l}\right)$.

One can show that these conditions are satisfied if

$\epsilon^{\rho}=-l \partial_{t} \zeta(t), \quad \epsilon^{t}=\zeta(t)+2 l^{2}\left(\mathrm{e}^{2 \rho / l}-f(t)\right)^{-1} \partial_{t}^{2} \zeta(t)$,

where $\zeta(t)$ is an arbitrary function of coordinate $t$. It is straightforward to find the transformation of the boundary 
metric under the diffeomorphism (43), namely,

$$
\begin{aligned}
\delta_{\epsilon} h_{t t}= & \mathrm{e}^{-2 \psi}\left(1-f(t) \mathrm{e}^{-2 \rho / l}\right) \\
& \times\left[\frac{1}{2} \partial_{t} f(t) \zeta(t)+f(t) \partial_{t} \zeta(t)-l^{2} \partial_{t}^{3} \zeta(t)\right] .
\end{aligned}
$$

The general transformation of the gauge fields $\mathcal{A}_{\mu}^{i}$ defined by

$\delta_{\epsilon} \mathcal{A}_{\mu}^{i}=\epsilon^{\lambda} \nabla_{\lambda} \mathcal{A}_{\mu}^{i}+\mathcal{A}_{\lambda}^{i} \nabla_{\mu} \epsilon^{\lambda}$

also leads to the following transformation: under the same diffeomorphism,

$\delta_{\epsilon} \mathcal{A}_{\rho}^{i}=-2 \lambda^{i} \mathrm{e}^{-\rho / l}\left(1+\sqrt{f(t)} \mathrm{e}^{-\rho / l}\right)^{-2} \partial_{t}^{2} \zeta(t)$.

To restore the gauge condition $\mathcal{A}_{\rho}^{(i)}=0$ (25) one should compensate for the diffeomorphism with a gauge transformations for each of the gauge fields as

$\mathcal{A}_{\mu}^{(i)} \rightarrow \mathcal{A}_{\mu}^{(i)}+\partial_{\mu} \Lambda^{(i)}$,

with gauge functions

$\Lambda^{i}=-2 l \lambda^{i} \mathrm{e}^{-\rho / l}\left(1+\sqrt{f(t)} \mathrm{e}^{-\rho / l}\right)^{-1} \partial_{t}^{2} \zeta(t)$.

Therefore, the combination of the allowed diffeomorphism (43) and the two gauge transformations (47) satisfy the gauge condition $(25){ }^{3}$

$\delta_{\epsilon+\Lambda^{i^{\prime}}} \mathcal{A}_{\rho}^{i^{\prime}}=\delta_{\epsilon} \mathcal{A}_{\rho}^{i}+\partial_{\rho} \Lambda_{\rho}^{i}=0$.

One can easily show that under the combination of the transformations the variations of the gauge fields are

$$
\begin{aligned}
& \delta_{\epsilon+\Lambda^{i^{\prime}}} \mathcal{A}_{t}^{i^{\prime}}=\frac{\lambda^{i}}{l}\left[\mathrm { e } ^ { - \rho / l } \left(\frac{1}{2} \partial_{t} f(t) \zeta(t)\right.\right. \\
& \left.\left.\quad+f(t) \partial_{t} \zeta(t)-l^{2} \partial_{t}^{3} \zeta(t)\right)-\partial_{t}(\zeta(t) \sqrt{f(t)})\right] .
\end{aligned}
$$

Let us emphasize that the gauge condition (25) can be satisfied if and only if the gauge transformations of both of the gauge fields (50) compensate for the diffeomorphism (43) and one cannot turn off one of them consistently.

From the 5D point of view this means that the rotating coordinates must play the same role in the asymptotic behavior of the metric. In other words, this implies that the boundary conditions of rotating coordinates, which determine the fluctuations of the associated components of the metric, should be of the same order. This is in precise agreement with the result of [10], which is reviewed in Sect. 2.

3 There is no summation over primed indices, in our notation.

\section{Conserved charges and central charge}

Now we want to investigate the asymptotic symmetries by employing the associated conserved charges. Since we are interested in the boundary energy-momentum tensor of a solution there are some subtleties we meet with as we define the associated conserved charges.

As shown in [17], the generators of the asymptotic symmetries are determined via the counter-term subtraction method (CTSM). These charges can differ from those defined usually. This method is based on the Peierls bracket [16], which has a covariant construction and is equivalent to the Poisson bracket on the space of observables. The charge calculated in this way is called the counter-term subtraction charge (CTSC), which is given by

$Q_{\xi}=-\delta_{G, \xi} S$

where $\xi$ is an infinitesimal transformation parameter and $G$ is a regular function such that near the past boundary $G=0$ and near the future boundary $G=1$.

As we already mentioned, we focus on the boundary fields and the boundary energy-momentum tensor. So, we need to determine the associated transformations. Using the induced transformation of an arbitrary boundary field, $\Phi$ is defined by $^{4}$

$\delta_{G, \xi} \Phi \equiv\left(\delta_{G \xi}-G \delta_{\xi}\right) \Phi$.

We will study diffeomorphism and gauge transformation charges in the following subsections.

\subsection{Diffeomorphism charge}

Under a general diffeomorphism transformation $x^{\mu} \rightarrow x^{\mu}+$ $\epsilon^{\mu}$ the variation of the full action is given by

$\delta_{\epsilon} S=\frac{1}{2} \int \mathrm{d} t \sqrt{-h} T^{a b} \delta_{\epsilon} \gamma_{a b}+\int \mathrm{d} t \sqrt{-h} J_{i}^{a} \delta_{\epsilon} \mathcal{A}_{a}^{i}+($ e.o.m)

where

$T_{t t}=-\frac{\pi \alpha}{4 G_{5}}\left(\frac{1}{l} \mathrm{e}^{-2 \psi} h_{t t}+\frac{2}{l} \mathrm{e}^{-4 \psi} M_{i j} \mathcal{A}_{t}^{i} \mathcal{A}_{t}^{j}\right)$,

$J_{i, t}=\frac{\pi \alpha}{G_{5}} M_{i j}\left(-n^{\mu} \mathcal{F}_{\mu t}^{j} \mathrm{e}^{-\psi}+\frac{1}{l} \mathcal{A}_{t}^{j}\right) \mathrm{e}^{-4 \psi}$,

$\delta_{\epsilon} \gamma_{a b}=\nabla_{a} \epsilon_{b}+\nabla_{b} \epsilon_{a}$,

$\delta_{\epsilon} \mathcal{A}_{a}^{i}=\epsilon^{b} \nabla_{b} \mathcal{A}_{a}^{i}+\mathcal{A}_{a}^{i} \nabla_{a} \epsilon^{b}$.

\footnotetext{
4 For details one can see [17].
} 
For an induced transformation (52) the variation of the full action is simplified:

$$
\begin{aligned}
\delta_{G, \epsilon} S= & \int \mathrm{d} t \sqrt{-h} T^{a b} \epsilon_{a} \nabla_{b} G+\int \mathrm{d} t \sqrt{-h} J_{i}^{a} \mathcal{A}_{b}^{i} \epsilon^{b} \nabla_{a} G \\
= & \sqrt{-h} h^{t t}\left(T_{t t}+J_{i, t} \mathcal{A}_{t}^{i}\right) \epsilon^{t} \\
& -\int \mathrm{d} t \sqrt{-h} \nabla_{a}\left[\left(T^{a b}+J_{i}^{a} \mathcal{A}^{i, b}\right) \epsilon_{b}\right] G \\
= & \sqrt{-h} h^{t t}\left(T_{t t}+J_{i, t} \mathcal{A}_{t}^{i}\right) \epsilon^{t}
\end{aligned}
$$

where in the last step we used the definition of the Noether charge associated to the Peierls bracket. Using the definition CTSC (51) the associated charge is given by

$Q_{\epsilon}=-\sqrt{-h} h^{t t}\left(T_{t t}+J_{i, t} \mathcal{A}_{t}^{i}\right)$.

As we can see, the energy-momentum tensor and both of the gauge fields appear in the diffeomorphism generator. One should note that, although the energy-momentum tensor $T_{t}$ and the $U(1)$ currents $J_{i, t}$ diverge as $\rho \rightarrow \infty$, the above combination is asymptotically finite, namely

$T_{t t}+J_{i, t} \mathcal{A}_{t}^{i}=\frac{\pi \alpha}{4 G_{5}} \mathrm{e}^{-4 \psi}\left(\frac{f(t)}{l}+\mathcal{O}\left(\mathrm{e}^{-\rho / l}\right)\right)$.

Note that for the extremal solution where $f(t)=0$ all nonextremal excitations will vanish. This is a consistency condition, since it implies that the excitations considered above keep the solution in the extremal limit [2].

\subsection{Gauge transformation charges}

Again one can explore the variation of the action under a gauge transformation $\delta_{\Lambda} \mathcal{A}_{a}^{i}=\partial_{a} \Lambda^{i}$ by using the CTSM, which is given by

$$
\begin{aligned}
\delta_{G, \Lambda^{i}} S & =\int \mathrm{d} t \sqrt{-h} \mathcal{J}_{i^{\prime}} \Lambda^{i^{\prime}} \partial_{a} G \\
& =\sqrt{-h} \mathcal{J}_{i^{\prime}}^{t} \Lambda^{i^{\prime}}-\int \mathrm{d} t \partial_{a}\left(\sqrt{-h} \mathcal{J}_{i^{\prime}}^{t} \Lambda^{i^{\prime}}\right) G,
\end{aligned}
$$

where

$\mathcal{J}_{i}^{a}=\frac{\pi \alpha}{G_{5} l} \mathrm{e}^{-4 \psi} M_{i j} \mathcal{A}^{j, a}$.

Similar to (58), the second term in (61) vanishes, and by definition the charges of the gauge transformations are given by

$Q_{\Lambda^{i}}=-\sqrt{-h} \mathcal{J}_{i}^{t}=-\frac{\pi \alpha}{G_{5} l} \mathrm{e}^{-4 \psi} \sqrt{-h} h^{t t} M_{i j} \mathcal{A}_{t}^{j}$.

Using the asymptotic behavior of the gauge fields and metric (29) it is easy to show that

$Q_{\Lambda^{i}}=\frac{\pi \alpha}{G_{5} l} \mathrm{e}^{-3 \psi} M_{i j} \lambda^{j}\left(1-2 \sqrt{f(t)} \mathrm{e}^{-\rho / l}+\mathcal{O}\left(\mathrm{e}^{-2 \rho / l}\right)\right)$.
For the near-horizon of extremal solutions the gauge transformation charges are given by

$Q_{\Lambda^{i}}=\frac{\pi \alpha}{G_{5} l} M_{i j} \lambda^{j}$

For the 4D extremal Kerr solution it was shown that this charge equals the angular momentum in the $4 \mathrm{D}$ point of view [13].

\subsection{Central charge}

Now we can explore the combination of the physical generators. Moreover, we can derive the central charge associated to the asymptotic transformations constructed in Sects. 4.1 and 4.2 .

The combined generator is given by

$Q_{\left(\epsilon+\Lambda^{1}+\Lambda^{2}\right)}=Q_{\epsilon} \epsilon+Q_{\Lambda^{i}} \Lambda^{i}$.

To study the transformations of this charge, it is natural to relate the transformation parameters, $\epsilon$ and $\Lambda^{i}$, to each other to treat the combined charge as a charge with one transformation parameter. The asymptotic behaviors of the transformation parameters are given by

$$
\begin{aligned}
\epsilon^{t} & =\zeta(t)+2 l^{2} \mathrm{e}^{-2 \rho / l} \partial_{t}^{2} \zeta(t)+\cdots, \\
\Lambda^{i} & =-2 l \lambda^{i} \mathrm{e}^{-\rho / l} \partial_{t}^{2} \zeta(t)+\cdots,
\end{aligned}
$$

and up to leading order one can write ${ }^{5}$

$\Lambda^{i}=l \mathcal{A}_{a}^{i} \partial_{\rho} \epsilon^{a}+\cdots$.

Thus, the gauge transformation part of the combined charge (66) becomes

$Q_{\Lambda^{i}} \Lambda^{i}=\sqrt{-h} h^{t t} \mathcal{A}_{t, i} \mathcal{J}_{t}^{i} \epsilon^{t}+\cdots$.

By employing Eq. (69), it is easy to see that the zeroth order of $\epsilon^{t}$ does not appear in (70) and the first term is $\mathrm{e}^{-2 \rho / l}$.

Now we are able to study the transformations of the combined charge given in (66). First we calculate the transformation of the diffeomorphism part of the combined charge, $T_{t t}+J_{t, i} \mathcal{A}_{t}^{i}$, which is asymptotically given by

$$
\begin{aligned}
& \delta_{\epsilon+\Lambda^{1}+\Lambda^{2}}\left(T_{t t}+J_{t, i} \mathcal{A}_{t}^{i}\right)=2\left(T_{t t}+J_{t, i} \mathcal{A}_{t}^{i}\right) \partial_{t} \zeta(t) \\
& \quad+\partial_{t}\left(T_{t t}+J_{t, i} \mathcal{A}_{t}^{i}\right) \zeta(t)+\mathcal{O}\left(\mathrm{e}^{-\rho / l}\right) .
\end{aligned}
$$

On the other hand, the variation of the gauge transformation part of the combined charge (66) is asymptotically

$\delta_{\epsilon+\Lambda^{1}+\Lambda^{2}}\left(\mathcal{J}_{t, i} \mathcal{A}_{t}^{i}\right)=\partial_{t}\left(\mathcal{J}_{t, i} \mathcal{A}_{t}^{i}\right) \zeta(t)-\frac{\pi \alpha l \mathrm{e}^{-4 \psi}}{2 G_{5}} \partial_{t}^{3} \zeta(t)$.

It seems that the weight of this part is zero but, as discussed in [13], we do not worry about this fact. As mentioned above

$\overline{5}$ There are some subtleties in this relation which are discussed in [13]. 
for this part of the combined charge, the asymptotic behavior is $\epsilon^{t} \sim \mathrm{e}^{-2 \rho / l}$ and it has effectively weight two.

Since the $\mathrm{AdS}_{2}$ radius $l_{\mathrm{AdS}}$ is dimensionless in the standard normalization of the transformation of the energymomentum tensor, the central charge is obtained via

$\delta T_{a b}=2 T_{a b} \partial_{t} \zeta(t)+\zeta(t) \partial_{t} T_{a b}-\frac{c}{12} \partial_{t}^{3} \zeta(t)$.

Thus one can read off the associated central charge by plugging (24), (71), and (72) into (73), which is namely given by

$c=\frac{6 \pi \alpha}{G_{5} \sqrt{\beta}} \mathrm{e}^{-4 \psi}$.

This is the associated central charge for a chiral CFT dual to the $\mathrm{AdS}_{2}$ geometry in the presence two gauge fields.

\subsection{Levels}

For completeness, we can find the level $k$ of the $U$ (1) gauge transformation which is defined by

$\delta_{\Lambda} J_{t}=\frac{k}{2} \partial_{t} \Lambda$

For the currents (55) associated to the gauge transformation of the $U(1)$ charges one can derive the level by

$\delta_{\Lambda^{i^{\prime}}} J_{t}^{i^{\prime}}=\frac{\pi \alpha l}{G_{5}} \mathrm{e}^{-4 \psi} \partial_{t} \Lambda^{i}$

By using (24) it is easy to show that the levels are determined by

$k^{i}=\frac{\pi \alpha}{G_{5} \sqrt{\beta}} \mathrm{e}^{-4 \psi}$.

Thus, the two gauge fields have the same level, namely

$k^{1}=k^{2}=c / 6$.

This relation between the central charge and the level of the $\mathrm{R}$-currents is similar to the results of $[13,26]$.

\section{Myers-Perry black hole}

As an example of our results we study the Myers-Perry black holes, which are a simple 5D solution with two angular momenta in the near-horizon geometry. The near-horizon geometry of the Myers-Perry black holes are of the form (6). Without loss of generality we assume that $0<a<b$, where $a$ and $b$ are the two parameters of the Myers-Perry black hole which are related to the two angular momenta. The parameters and functions of the metric (6) for this solution are given by Myers and Perry [15]
$F(\theta)=\frac{\sigma(\theta)}{4}, \quad \sigma(\theta)=a b+a^{2} \cos ^{2} \theta+b^{2} \sin ^{2} \theta$,

$k^{i} \frac{\partial}{\partial x^{i}}=\frac{2 \sqrt{a b}}{a(a+b)^{2}} \frac{\partial}{\partial \phi}+\frac{2 \sqrt{a b}}{b(a+b)^{2}} \frac{\partial}{\partial \psi}$,

$f_{\phi \phi}(\theta)=\frac{(a+b)^{2}}{\sigma(\theta)^{2}} a \sin ^{2} \theta\left(a+b \sin ^{2} \theta\right)$,

$f_{\psi \psi}(\theta)=\frac{(a+b)^{2}}{\sigma(\theta)^{2}} b \cos ^{2} \theta\left(b+a \cos ^{2} \theta\right)$,

$f_{\phi \psi}(\theta)=\frac{(a+b)^{2}}{\sigma(\theta)^{2}} a b \sin ^{2} \theta \cos ^{2} \theta$.

Considering these expansions one can calculate the parameters (16) and (17) appeared in the central charge (74) and the levels (77) as

$\alpha=2(a+b)^{2} \sqrt{a b}, \quad \beta=\frac{4 a b}{(a+b)^{2}}$.

One can see from (16) that the parameter $\alpha$ is proportional to the area of the horizon which is essentially the Wald entropy of the black hole. So it is natural to study the symmetries of the rotating coordinates, which do not affect the entropy. For general study of this argument one can see [10]. The geometry of the Myers-Perry black hole (83) has a symmetry under $a \leftrightarrow b$ compensating for $\theta \rightarrow \pi / 2-\theta$. Thus after integrating over angular coordinates one would expect that the central charge (74) has this symmetry. Using (74), (78), and (84) the central charge and levels are given by

$c=\frac{3}{2} \pi(a+b)^{3}, \quad k_{\phi}=k_{\psi}=\frac{1}{4} \pi(a+b)^{3}$

This is a sum of two central charges derived in [4]. It is easy to show that, for Myers-Perry black holes, this is in perfect agreement with the results obtained from five-dimensional point of view reviewed in Sect. 2 and is given in Eq. (5).

\section{Conclusion and discussion}

In this paper we studied the near-horizon geometry of the $5 \mathrm{D}$ extremal rotating black holes from the $2 \mathrm{D}$ point of view by reducing the theory over the angular part of the coordinates following [13]. We showed that the consistency of the boundary conditions implies that both of the gauge fields, which correspond to two angular momenta in 5D, appear in the same manner. By studying the variation of the boundary energy-momentum tensor we calculate the central charge of the CFT dual to the reduced solution which has a $\mathrm{AdS}_{2}$ geometry.

Although we did not trace the power of the fluctuations of the $5 \mathrm{D}$ metric due to the process of reduction, we showed that for consistent boundary conditions the results are in agreement with the calculations of 5D viewpoint [10]. The advantage of the consistency is that they compensate for the vari- 
ations of both of the gauge fields with the diffeomorphism variation studied in Sect. 3.4. It is interesting to study the relation between consistency of boundary conditions from 5D and 2D point of views which are given in (2) and (42), respectively.

Finally, we conclude that from a 2D point of view that the consistency of the boundary conditions respects the symmetry of the near-horizon geometry discussed in [10] in the Kerr/CFT approach. Since the parameter $\alpha$ appearing in the central charge (74) is proportional to the Wald entropy (16), it is natural to study the effect of the symmetry of the nearhorizon geometry. The symmetry of the rotating coordinates in the 5D point of view inherited by $M_{i j}$, see (18), and its determinant. The result of this symmetry in the $5 \mathrm{D}$ perspective was investigated in [10], but it is not realized in the Myers-Perry black holes studied in Sect. 5.

The reduction of extremal black holes in higher dimensions to 2D is also the basic argument of Sen's quantum entropy function [12]. This reduction produce an $\mathrm{AdS}_{2}$ metric, some scalar fields and a number of gauge fields associated to the angular momenta in higher dimensions. In [12] it was shown that the thermodynamics of such solutions are determined from quantum entropy function which is defined by

$d_{\text {hor }}(\vec{q})=\left\langle\exp \left[-i q_{i} \oint \mathrm{d} \theta A_{\theta}^{i}\right]\right\rangle_{\mathrm{AdS}_{2}}^{\text {finite }}$

in the euclidean frame. Here $\oint \mathrm{d} \theta A_{\theta}^{i}$ denotes the integral of $i$ th gauge field along the boundary of $\mathrm{AdS}_{2}$ and $q_{i}$ is the $i$ th electric charge. It is clear that all of the gauge fields play the same role in the thermodynamics of the reduced extremal solution. So, it is natural to expect that there is one CFT which is corresponded to the near-horizon geometry of an ERBH. This was discussed for 5D ERBH in [10] by employing the Kerr/CFT approach and here we confirm the results from 2D point of view. It is worth to study the relation between these approaches and Sen's quantum entropy function.

Although we have not studied higher-dimensional extremal black holes we expect that one can apply this formalism in those cases and after the reduction on angular coordinates, all of the gauge fields play the same role from $2 \mathrm{D}$ perspective and there is only one CFT corresponding to the near-horizon geometry of extremal black holes.

Recently it was proposed a systematic method for deriving the order of the boundary conditions of the metric for topologically massive gravity [27]. Another interesting question is the extension of this method to higher dimensions and compare our results with this extension.

In this paper we limited ourself to the solutions of 5D Einstein gravity but one can generalize this method to the solutions of other gravity theory e.g. supergravity. As a simple example with only one rotating coordinate one can study supersymmetric black ring [28]. Microscopic description of this solution is studied using other methods in $[3,29,30]$.

Acknowledgments I would like to thank Farhang Loran for useful discussions and comments on the draft and Department of Physics of Isfahan University of Technology where I did my Ph.D. and part of this work was done. This work is supported by Foundation for Polish Science MPD Programme co-financed by the European Regional Development Fund, agreement No. MPD/2009/6.

Open Access This article is distributed under the terms of the Creative Commons Attribution License which permits any use, distribution, and reproduction in any medium, provided the original author(s) and the source are credited.

Funded by $\mathrm{SCOAP}^{3}$ / License Version CC BY 4.0.

\section{References}

1. A. Strominger, C. Vafa, Microscopic origin of the BekensteinHawking entropy. Phys. Lett. B 379, 99 (1996). [hep-th/9601029]

2. M. Guica, T. Hartman, W. Song, A. Strominger, The Kerr/CFT Correspondence. Phys. Rev. D 80, 124008 (2009). [arXiv:0809. 4266 [hep-th]]

3. F. Loran, H. Soltanpanahi, Near the horizon of 5D black rings. JHEP 0903, 035 (2009). [arXiv:0810.2620 [hep-th]]

4. H. Lu, J. Mei, C.N. Pope, Kerr/CFT correspondence in diverse dimensions. JHEP 0904, 054 (2009). [arXiv:0811.2225 [hep-th]]

5. H. Isono, T.-S. Tai, W.-Y. Wen, Kerr/CFT correspondence and fivedimensional BMPV black holes. Int. J. Mod. Phys. A 24, 5659 (2009). [arXiv:0812.4440 [hep-th]]

6. T. Azeyanagi, N. Ogawa, S. Terashima, Holographic duals of Kaluza-Klein black holes. JHEP 0904, 061 (2009). [arXiv:0811. 4177 [hep-th]]

7. D.D.K. Chow, M. Cvetic, H. Lu, C.N. Pope, Extremal black hole/CFT correspondence in (gauged) supergravities. Phys. Rev. D 79, 084018 (2009). [arXiv:0812.2918 [hep-th]]

8. Y. Nakayama, Emerging AdS from extremally rotating NS5branes. Phys. Lett. B 673, 272 (2009). [arXiv:0812.2234 [hep-th]]

9. T. Azeyanagi, N. Ogawa, S. Terashima, The Kerr/CFT correspondence and string theory. Phys. Rev. D 79, 106009 (2009). [arXiv: 0812.4883 [hep-th]]

10. F. Loran, H. Soltanpanahi, 5D extremal rotating black holes and CFT duals. Class. Quant. Grav. 26, 155019 (2009). [arXiv:0901. 1595 [hep-th]]

11. D. Astefanesei, Y.K. Srivastava, CFT duals for attractor horizons. Nucl. Phys. B 822, 283 (2009). [arXiv:0902.4033 [hep-th]]

12. A. Sen, Quantum entropy function from $\operatorname{AdS}(2) / C F T(1)$ correspondence. Int. J. Mod. Phys. A 24, 4225 (2009). [arXiv:0809.3304 [hep-th]]

13. A. Castro, F. Larsen, Near extremal Kerr entropy from $\operatorname{AdS}(2)$ quantum gravity. JHEP 0912, 037 (2009). [arXiv:0908.1121 [hepth]]

14. T. Hartman, A. Strominger, Central charge for $\operatorname{AdS}(2)$ quantum gravity. JHEP 0904, 026 (2009). [arXiv:0803.3621 [hep-th]]

15. R.C. Myers, M.J. Perry, Black holes in higher dimensional spacetimes. Ann. Phys. 172, 304 (1986)

16. R.E. Peierls, The commutation laws of relativistic field theory. Proc. R. Soc. Lond. A 214, 143 (1952)

17. S. Hollands, A. Ishibashi, D. Marolf, Counter-term charges generate bulk symmetries. Phys. Rev. D 72, 104025 (2005). [hep-th/ 0503105]

18. D. Astefanesei, K. Goldstein, R.P. Jena, A. Sen, S.P. Trivedi, Rotating attractors. JHEP 0610, 058 (2006). [hep-th/0606244] 
19. H.K. Kunduri, J. Lucietti, H.S. Reall, Near-horizon symmetries of extremal black holes. Class. Quant. Grav. 24, 4169 (2007). [arXiv: 0705.4214 [hep-th]]

20. D. Astefanesei, H. Yavartanoo, Stationary black holes and attractor mechanism. Nucl. Phys. B 794, 13 (2008). [arXiv:0706.1847 [hepth]]

21. G. Barnich, F. Brandt, Covariant theory of asymptotic symmetries, conservation laws and central charges. Nucl. Phys. B 633, 3 (2002). [hep-th/0111246]

22. G. Barnich, G. Compere, Surface charge algebra in gauge theories and thermodynamic integrability. J. Math. Phys. 49, 042901 (2008). [arXiv:0708.2378 [gr-qc]]

23. J.D. Brown, M. Henneaux, Central charges in the canonical realization of asymptotic symmetries: an example from three-dimensional gravity. Commun. Math. Phys. 104, 207 (1986)

24. D. Grumiller, W. Kummer, D.V. Vassilevich, Dilaton gravity in two-dimensions. Phys. Rep. 369, 327 (2002). [hep-th/0204253]
25. D. Grumiller, R. Meyer, Ramifications of lineland. Turk. J. Phys. 30, 349 (2006). [hep-th/0604049]

26. A. Castro, D. Grumiller, F. Larsen, R. McNees, Holographic description of AdS(2) black holes. JHEP 0811, 052 (2008). [arXiv: 0809.4264 [hep-th]]

27. K. Skenderis, M. Taylor, B.C. van Rees, AdS boundary conditions and the topologically massive gravity/CFT correspondence. [arXiv: 0909.5617 [hep-th]]

28. H. Elvang, R. Emparan, D. Mateos, H.S. Reall, A supersymmetric black ring. Phys. Rev. Lett. 93, 211302 (2004). [hep-th/0407065]

29. M. Cyrier, M. Guica, D. Mateos, A. Strominger, Microscopic entropy of the black ring. Phys. Rev. Lett. 94, 191601 (2005). [hep-th/0411187]

30. R. Emparan, H.S. Reall, Black rings. Class. Quant. Grav. 23, R169 (2006). [hep-th/0608012] 